\title{
SYNTHESIS OF A MULTI-VALUED DIAGNOSTIC MODEL USING THE WIGNER-VILLE TRANSFORMATION
}

\section{SYNTEZA WIELOWARTOŚCIOWEGO MODELU DIAGNOSTYCZNEGO Z WYKORZYSTANIEM TRANSFORMACJI WIGNER-VILLE'a}

\author{
Henryk Borowczyk
}

Air Force Institute of Technology, Instytut Techniczny Wojsk Lotniczych

\begin{abstract}
The paper presents a method of creating a multi-valued diagnostic model using Wigner-Ville transformation of vibroacoustic signals measured on a real object. The result is a transformation matrix in which the rows correspond to the frequencies $F$ and the column - times T of successive samples of the signal. Integer coding involves assigning values to the coefficient specified range of real numbers one integer. After applying the coding a multi-valued diagnostic model is obtained. The model describes the relationship between a set of significant frequencies, rms values of Wigner-Ville transform's coefficients, and a set of diagnosed object failures.
\end{abstract}

Keywords: multi-valued diagnostic model, Wigner-Ville transform, signal parameterization

Streszczenie: $W$ pracy przedstawiono metode tworzenia wielowartościowego modelu diagnostycznego z wykorzystaniem transformacji Wigner-Ville'a sygnatów wibroakustycznych zmierzonych na obiekcie rzeczywistym. Wynikiem transformacji jest macierz WV, której wiersze odpowiadaja częstotliwościom F, a kolumny czasom $T$ pobrania kolejnych próbek sygnatu. Kodowanie catkowitoliczbowe polega na przypisaniu wartościom wspótczynników z określonego przedziału liczb rzeczywistych jednej liczby catkowitej ze znakiem. Po zastosowaniu kodowania uzyskuje się wielowartościowy model diagnostyczny opisujacy relacje między zbiorem częstotliwości istotnych, wartościami skutecznymi wspótczynników transformaty Wigner-Ville'a i zbiorem stanów diagnozowanego obiektu.

Stowa kluczowe: wielowartosciowy model diagnostyczny, transformacja WignerVille'a, parametryzacja sygnału 
Synthesis of a multi-valued diagnostic model using the Wigner-Ville transformation Synteza wielowartościowego modelu diagnostycznego z wykorzystaniem...

\section{SYNTHESIS OF A MULTI-VALUED DIAGNOSTIC MODEL USING THE WIGNER-VILLE TRANSFORMATION}

\section{Introduction}

Vibrations and noise are used as information carriers on the technical condition of objects [6-9, 14]. Information necessary in the diagnosis process is obtained through active and passive experiments conducted on real objects, laboratory tests and simulations and expert knowledge [6, 9-14, 17].

In order to extract information about dynamic processes related to the functioning of the object and the impact of the damage, the signals are transformed into the frequency domain $[8,9]$.

The paper presents the method of synthesizing a multivalued diagnostic model using the results of an experiment conducted on a real object [18] and Wigner-Ville transformation $[1,2,16]$.

Its essence is to determine the matrix of WV transform coefficients, calculate the root mean square level of the coefficients for the individual frequencies, and then determine the set of significant frequencies based on the local maxima of the root mean square level values.

\section{Diagnostic experiment $[4,5]$}

In the diagnosis of complex technical objects, the following types of experiments can be distinguished $[4,5]$ :

- Research - defining the value of symptoms $d_{r} \in D, r=1,2, \ldots, t$ for known object states $e_{i} \in E, i=1,2, \ldots, n$, which enables the development of a diagnostic model - relations between states and symptoms;

- Operational - determine the current value of the symptoms, and then determine the current state of the object using the diagnostic model;

- Composite - combines the features of an operational and research experiment.

In this work we will consider an experiment Wibr (g) in vibroacoustic diagnostics. In the first stage the test is carried out on the fixed operating point of the object. The research experiment $\mathrm{Wibr}_{B}(\mathrm{~g})$ may be presented in the following form:

$$
W_{i b r}(z a k r)=\left\langle E_{B W i b r}(z a k r),\left\{Y_{B W i b r}(z a k r)\right\}\right\rangle
$$

where:

- $E_{B W i b r}(z a k r)$ - a set of introduced defects of the elements;

- $Y_{B W i b r}(z a k r)$ - a set of vibroacoustic diagnostic signals;

- zakr - object operating point.

The operational experiment is carried out on a real object of unknown technical condition.

$$
W_{i b r}(z a k r)=\left\langle e_{\text {OWibr }}^{?}(z a k r), Y_{\text {OWibr }}(z a k r)\right\rangle
$$


If the results of the diagnostic tests can be mathed up with knowledge base, ie:

$$
Y_{\text {OWibr }}(z a k r) \subseteq\left\{Y_{B W i b r}(z a k r)\right\}
$$

the current state of the object is determined.

$$
e_{\text {OWibr }}^{?}(z a k r)=\left(e_{B W i b r}^{i}(z a k r) \in E_{B W i b r}(z a k r)\right)
$$

Otherwise, the condition of the object remains unknown and the method of the composite experiment should be applied.

Composite testing consists of two phases: operational and research, and mandatory is the operational phase. If the symptoms from the current diagnostic test are consistent with the current knowledge, the state of the object is identified. Otherwise, a test phase is started, which updates diagnostic knowledge using information from the operating phase, recovery process, direct state assessment methods (including checkout disassembly) and simulation tests.

$$
W i b r_{Z}(z a k r)=\left\langle e_{Z W i b r}^{*}(z a k r), Y_{Z W i b r}(z a k r)\right\rangle
$$

If the relation is satisfied:

$$
Y_{\text {ZWibr }}(z a k r) \subseteq\left\{Y_{B W i b r}(z a k r)\right\}
$$

the current state is an element of a set of known states:

$$
e_{\text {ZWibr }}^{*}(z a k r)=\left(e_{B W i b r}^{i}(z a k r) \in E_{B W i b r}(z a k r)\right)
$$

if not, the knowledge base update procedure is started:

$$
\begin{gathered}
\left\{Y_{B W i b r}(z a k r)\right\}^{\prime}=\left\{Y_{B W i b r}(z a k r)\right\} \cup Y_{Z W i b r}(z a k r) \\
E_{B W i b r}^{\prime}(z a k r)=E_{B W i b r}(z a k r) \cup e_{Z W i b r}^{*}(z a k r)
\end{gathered}
$$

As a result, the cardinality of damage sets and the set of diagnostic signals included in the knowledge base increases.

An example of this is an experimental research project that covered issues related to the complex diagnosis of the Allison 250-C20B turbine engine bearing system [6].

\section{Parameterization of diagnostic signals using the Wigner-Ville transform}

The diagnostic test results are the sets of primary signals that should be processed to allow for effective diagnostic inference [6, 12, 13]. For this purpose, parameterization of the time series is performed by finding the representation in the form of a small set of parameters, while maintaining the diagnostic information necessary in the inference process $[3,6,10]$. 
Synthesis of a multi-valued diagnostic model using the Wigner-Ville transformation Synteza wielowartościowego modelu diagnostycznego z wykorzystaniem...

Vibroacoustic signals generated by rotor machines are transformed into the frequency domain, as well as time-frequency and time-scale. In this work, parameterization of vibroacoustic signals was performed using the Wigner-Ville transform $[1,2]$.

The Wigner-Ville transform generates a time-frequency representation of the signals in the form of the energy distribution of the signal relative to the two variables: time and frequency. The signal energy can be defined as the square of the signal module or its Fourier transform [2]:

$$
E_{x}=\int_{-\infty}^{+\infty}|x(t)|^{2} d t=\int_{-\infty}^{+\infty}|X(\omega)|^{2} d \omega
$$

By putting the total energy density in time and frequency one can save [2]

$$
E_{x}=\int_{-\infty}^{+\infty} \int_{-\infty}^{+\infty} \rho(t, \omega) d t d \omega
$$

Wigner-Ville distribution (WVD) is defined as follows [2]:

$$
W_{x}(t, \omega)=\int_{-\infty}^{+\infty} x(t+\tau / 2) x^{*}(t-\tau / 2) e^{-i 2 \pi \omega \tau} d \tau
$$

or

$$
W_{x}(t, \omega)=\int_{-\infty}^{+\infty} X(\omega+\xi / 2) x^{*}(t-\xi / 2) e^{i 2 \pi \xi \tau} d \xi
$$

\section{WVD distribution properties}

Energy conservation [2]

$$
E_{x}=\int_{-\infty}^{+\infty} \int_{-\infty}^{+\infty} W_{x}(t, \omega) d t d \omega
$$

Marginal properties [2]

$$
\begin{aligned}
& \int_{-\infty}^{+\infty} W_{x}(t, \omega) d t=|X(\omega)|^{2} \\
& \int_{-\infty}^{+\infty} W_{x}(t, \omega) d \omega=|x(t)|^{2}
\end{aligned}
$$

Takes values from a set of real numbers [2]

$$
W_{x}(t, \omega) \in \mathfrak{R}, \forall t, \omega
$$

Translation covariance [2]

$$
\begin{aligned}
& y(t)=x\left(t-t_{0}\right) \Rightarrow W_{y}(t, \omega)=W_{x}\left(t-t_{0}, \omega\right) \\
& y(t)=x(t) e^{j 2 \pi \omega_{0} t} \Rightarrow W_{y}(t, \omega)=W_{x}\left(t, \omega-\omega_{0}\right)
\end{aligned}
$$


Dilation covariance [2]

$$
y(t)=\sqrt{k} x(k t) ; k>0 \Rightarrow W_{y}(t, \omega)=W_{x}\left(k t, \frac{\omega}{k}\right)
$$

Compatibilty with filtering [2]

$$
y(t)=\int_{-\infty}^{+\infty} h(t-s) x(s) \mathrm{ds} \Rightarrow \int_{-\infty}^{+\infty} W_{h}(t-s, \omega) W_{x}(\mathrm{~s}, \omega) \mathrm{ds}
$$

Compatibilty with modulation [2]

$$
y(t)=m(t) x(t) \Rightarrow W_{y}(t, \omega)=\int_{-\infty}^{+\infty} W_{m}(t, \omega-\xi) W_{x}(\mathrm{~s}, \xi) \mathrm{d} \xi
$$

Practical use of the Wigner-Ville transform requires the use of appropriate software. Time-Frequency Toolbox (TFTb) for Matlab [16] was developed by teams from France (CNRS - Center National de la Recherche Scientifique) and USA (Rice University) [1, 2].

The following is a header of the Wigner-Ville transformation function [2].

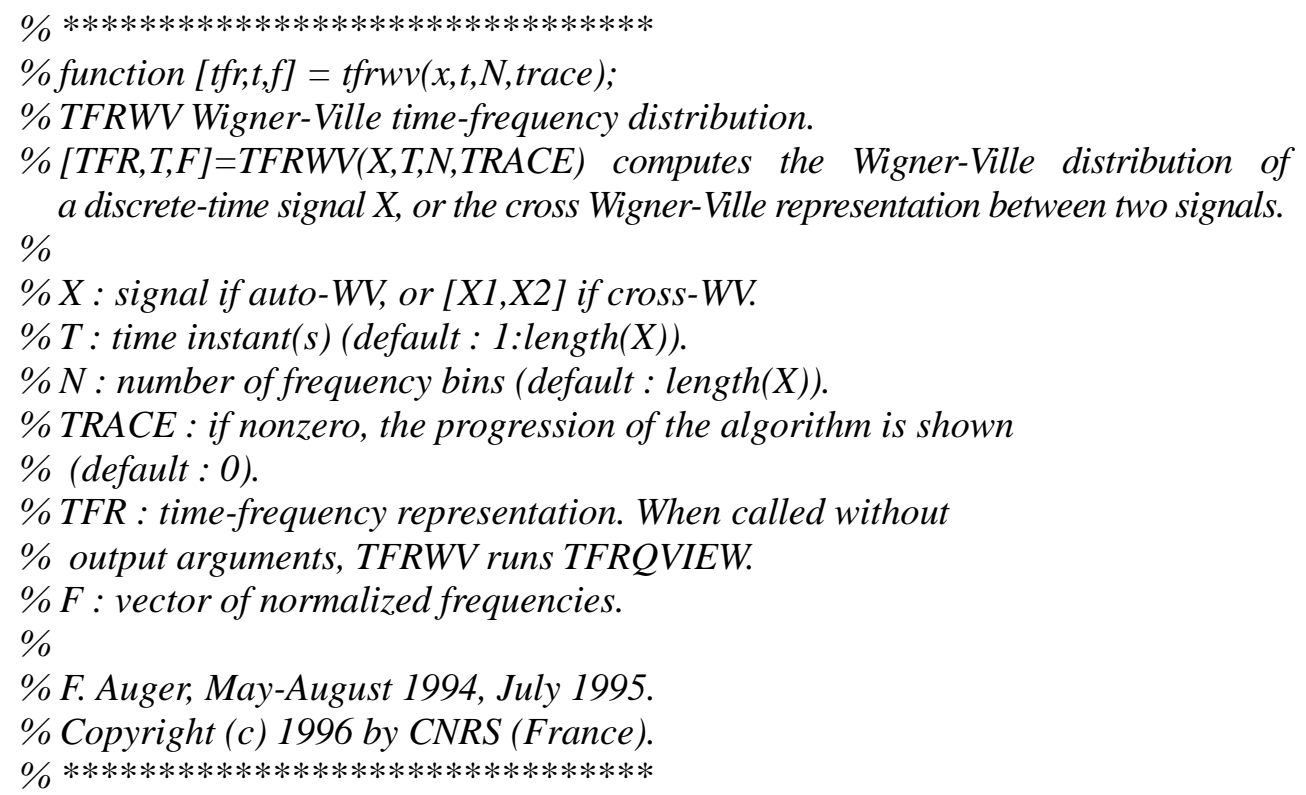

The input vectors are: $x$ - samples of the analyzed signal and $t$ - time of sampling $(N x)$ and assumed frequency resolution $N<=N x$.

The output parameters are: $t f r$ matrix of Wigner-Ville transform coefficients with dimensions $[N, N x]$ and frequency vector $f$ normalized with respect to sampling frequency $f_{s}$.

The obtained results provide the basis for determining the multivalued representation of the WV transform for implementation in the diagnostic model. 
Synthesis of a multi-valued diagnostic model using the Wigner-Ville transformation Synteza wielowartościowego modelu diagnostycznego z wykorzystaniem...

\section{Multivalued representation of the Wigner-Ville transform}

The algorithm of the proposed method for determining the multivalued representation of a WV transform consists of the following steps:

- determination of the Wigner-Ville transform of the analyzed signal (using the function $t f r w v$ described above);

- calculation of the root mean square level of the Wigner-Ville transform coefficients for the set of frequencies $F$ included in the original WV matrix;

- determine the set $F i$ of significant frequencies based on local maxima of WV transform coefficients' root mean square level;

- performing multivalent coding of root mean square level of WV transform coefficients corresponding to significant frequencies $F i$.

The key elements of the algorithm are illustrated by the following pseudo-code:

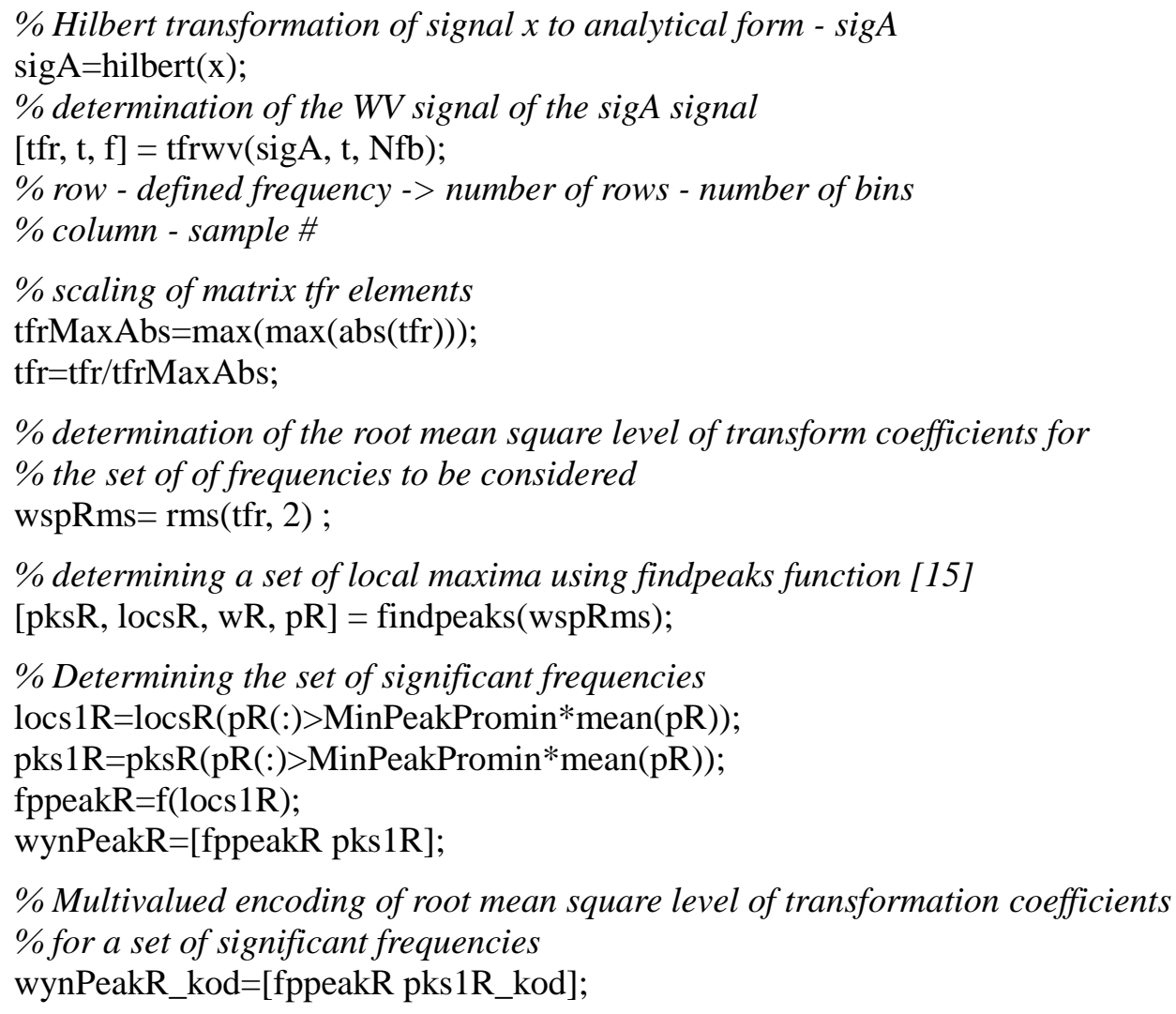

Multivalued encoding involves assigning the values of the coefficients from a given range of real numbers to one integer with a sign. The numerical range of coefficients should be divided into intervals so that, on the one hand, they provide the highest resolution (state/damage discrimination) and, on the other hand, minimize the number of false diagnoses $[4,5]$. 
Designing intervals can be considered as a formal issue of data clustering or heuristics. In the first attempt, the most often used is a heuristic approach that uses expert knowledge and does not require a large set of statistical data.

In the process of the diagnostic system development, after the accumulation of the appropriate amount of experimental material, the predefined intervals are subjected to periodic updating using formal methods $[4,5]$.

\section{An example of signal analysis}

The vibration signal (LL09A-2) measured on a real object was analyzed.

Fig. 1 shows the signal time waveform, spectral energy density and Wigner-Ville transform 3D plot.

Figure 2 shows:

- the root mean square level of the WV transform coefficients for all frequencies included in the tfr transform (continuous line);

- set of significant frequencies - stem plot.

Multivalued encoding of root mean square level of WV transform coefficients for significant frequencies is presented in Fig. 3.
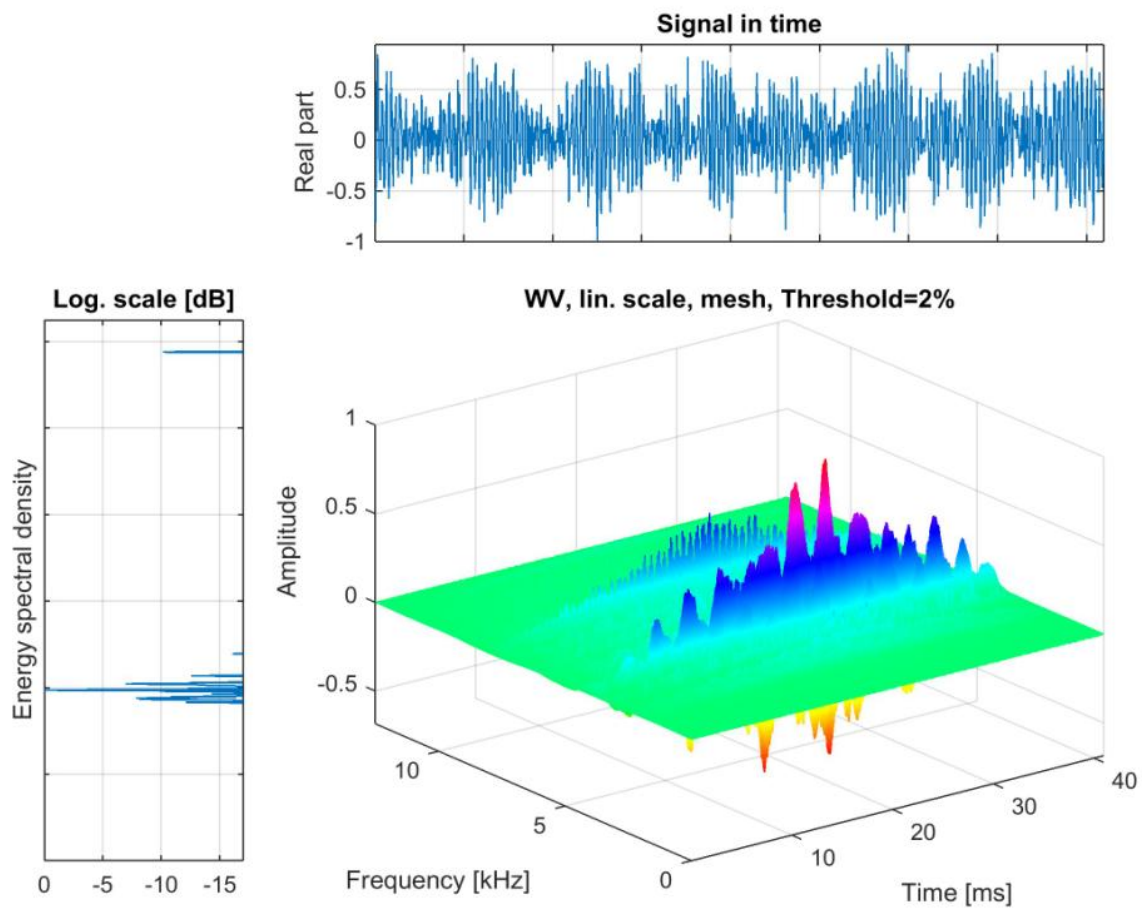

Fig. 1 Graphical representation of the Wigner-Ville transform of the analyzed signal 
Synthesis of a multi-valued diagnostic model using the Wigner-Ville transformation Synteza wielowartościowego modelu diagnostycznego z wykorzystaniem...

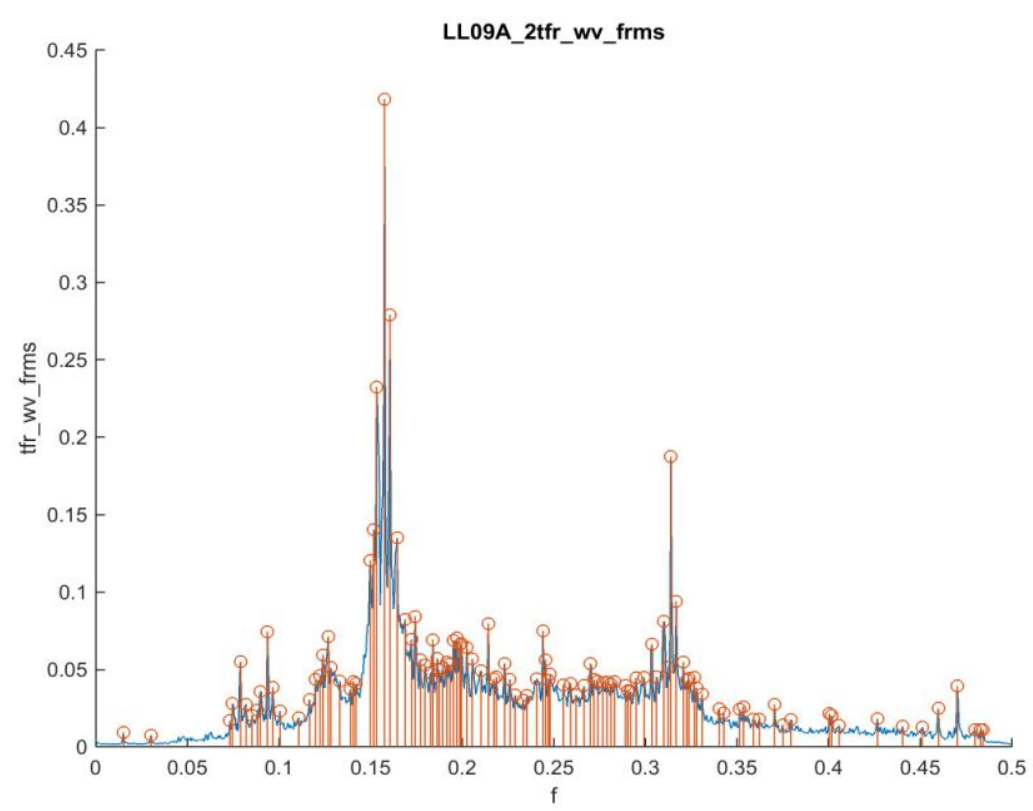

Fig. 2 Graphical representation of the significant frequency set of the analyzed signal

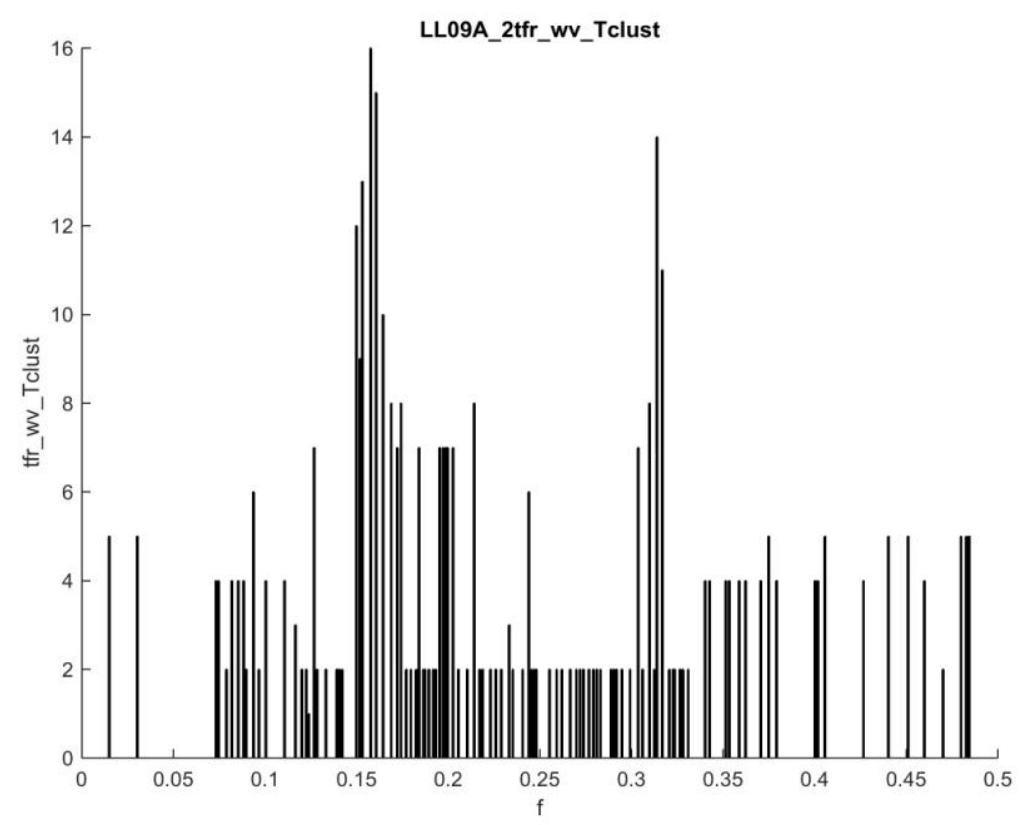

Fig. 3 Multivalued representation of root mean square level of WV transform coefficients for significant frequencies 


\section{Summary}

The paper presents the problem of synthesis of a diagnostic model using the Wigner-Ville transform of vibroacoustic signals measured on a real object.

Because diagnostic inferences are not based on point values of the characteristics, they have multi-valued coding. Signal analysis results provide a way to compare the diagnostic information obtained from the Wigner-Ville transform and to track changes in the technical condition of the facility during operation.

The results of the work can be used in expert diagnostic systems to automate the analysis.

\section{Bibliography}

[1] Auger, F., et al., Time-Frequency Toolbox. Reference Guide( CNRS - France, Rice University -USA). 1995-1996.

[2] Auger, F., et al., Time-Frequency Toolbox. User's Guide( CNRS - France, Rice University - USA). 1995-1996.

[3] Borowczyk, H., Ekspertowy system wspomagania wnioskowania diagnostycznego. W: Borowczyk H.. (red.) Problemy kompleksowego diagnozowania układu tożyskowania turbinowego silnika śmigłowcowego. Wyd. ITWL. Warszawa 2011. s. 147-163.

[4] Borowczyk, H., Elementy kompleksowej diagnostyki złożonych obiektów technicznych (monografia w przygotowaniu do druku).

[5] Borowczyk, H., A Multi-Valued Diagnostic Model Synthesis Based on Descrete Wavelet Transform, Journal of KONBiN 1 (17) 2011.https://doi.org/10.2478/jok-2013-0104.

[6] Borowczyk, H., ed. Problemy kompleksowego diagnozowania uktadu tożyskowania turbinowego silnika śmigłowcowego. Wyd. ITWL. Warszawa $2011163 \mathrm{~s}$.

[7] Borowczyk, H., P. Lindstedt, and J. Magier, Uktad tożyskowania turbinowego silnika śmigłowcowego jako obiekt diagnozowania. $W$ : Borowczyk H.. (red.) Problemy kompleksowego diagnozowania uktadu tożyskowania turbinowego silnika śmigłowcowego. Wyd. ITWL. Warszawa 2011. s. 21-30.

[8] Cempel, C., Podstawy wibroakustycznej diagnostyki maszyn, WNT, Warszawa 1982.

[9] Cempel, C., Wibroakustyka stosowana, WNT, Warszawa 1989.

[10] Cholewa, W., Data processing and reasoning in technical diagnostics. WNT, Warszawa, 1995.

[11] Korbicz, J., Diagnostyka procesów. Modele. Metody sztucznej inteligencji. Zastosowania. WNT, Warszawa 2002.

[12] Lindstedt, P., Praktyczna diagnostyka maszyn i jej teoretyczne podstawy. Wyd. Naukowe ASKON, Warszawa 2002.

[13] Lindstedt, P. and H. Borowczyk, Kompleksowy system diagnostyczny statku powietrznego, w: Problemy Badań i Eksploatacji Techniki Lotniczej, tom 4, rozdz. 7, Wyd. ITWL, Warszawa 1999. 
Synthesis of a multi-valued diagnostic model using the Wigner-Ville transformation Synteza wielowartościowego modelu diagnostycznego z wykorzystaniem...

[14] Lindstedt, P., H. Borowczyk, and J. Magier, Diagnostyka silnika śmigłowcowego $w$ inżynieryjno-lotniczym otoczeniu, Prace Naukowe ITWL, zeszyt nr 17. Wyd. ITWL, Warszawa 2003.

[15] MathWorks. findpeaks - help. Available from: www.mathworks.com/help/signal/ref/findpeaks.html?s tid=doc ta.

[16] MathWorks. Help Available from: https://www.mathworks.com/help/.

[17] Niziński, S., Elementy eksploatacji obiektów technicznych, Wyd. Uniwersytetu Warmińsko-Mazurskiego, Olsztyn 2000.

[18] Spychała, J., M. Szczekala, and M. Żokowski, Diagnozowanie $w$ dziedzinie wibroakustycznej. W: Borowczyk H.. (red.) Problemy kompleksowego diagnozowania układu tożyskowania turbinowego silnika śmigłowcowego. Rozdz. 5. Wyd. ITWL Warszawa 2011.

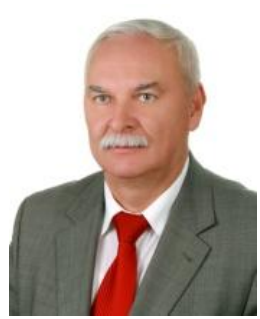

PhD Eng. Henryk Borowczyk, Aircraft Engine Division, Air Force Institute of Technologyl, Warsaw. Research activities: Comprehensive diagnostics of turbine aircraft engines using information theory, mathematical identification methods of dynamic systems and artificial intelligence. 


\section{METODA SYNTEZY WIELOWARTOŚCIOWEGO MODELU DIAGNOSTYCZNEGO Z WYKORZYSTANIEM TRANSFORMACJI WIGNER-VILLE'a}

\section{Wstęp}

W diagnostyce wibroakustycznej maszyn wirnikowych wykorzystuje się drgania i hałas jako nośniki informacji o stanie technicznym obiektów [6-9, 14]. Informacja niezbędna $\mathrm{w}$ procesie diagnozowania pozyskiwana jest $\mathrm{w}$ wyniku czynnych i biernych eksperymentów przeprowadzanych na obiekcie rzeczywistym, badań laboratoryjnych i na modelach symulacyjnych oraz kwerend wiedzy ekspertów $[6,9-14,17]$.

W celu wyodrębnienia informacji o procesach dynamicznych związanych z funkcjonowaniem obiektu oraz oddziaływaniem uszkodzeń, sygnały poddawane są transformacjom do dziedziny częstotliwości [8, 9].

W pracy przedstawiono metodę syntezy wielowartościowego modelu diagnostycznego z wykorzystaniem wyników eksperymentu przeprowadzonego na obiekcie rzeczywistym [18] oraz transformaty Wigner-Ville'a [1, 2, 16].

Jej istota polega na wyznaczeniu macierzy współczynników transformaty WV, obliczeniu wartości skutecznych współczynników poszczególnych częstotliwości, a następnie określeniu zbioru częstotliwości istotnych na podstawie lokalnych maksimów wartości skutecznych.

\section{Eksperyment diagnostyczny $[4,5]$}

W diagnostyce złożonych obiektów technicznych można wyróżnić następujące rodzaje eksperymentów $[4,5]$ :

- badawczy - określenie wartości symptomów $d_{r} \in D, r=1,2, \ldots, t$ dla znanych stanów obiektu $e_{i} \in E, i=1,2, \ldots, n$, co umożliwia zbudowanie modelu diagnostycznego - relacji między stanami a symptomami;

- operacyjny - określenie bieżących wartości symptomów, a następnie ustalenie aktualnego stanu obiektu z wykorzystaniem modelu diagnostycznego;

- zespolony - łączy cechy eksperymentu operacyjnego i badawczego.

W niniejszej pracy rozpatrywany będzie eksperyment w diagnostyce wibroakustycznej $\operatorname{Wibr}(\mathrm{g})$. W pierwszym etapie badania prowadzone są na ustalonych zakresach pracy obiektu. 
Synthesis of a multi-valued diagnostic model using the Wigner-Ville transformation Synteza wielowartościowego modelu diagnostycznego z wykorzystaniem...

Eksperyment badawczy $\operatorname{Wibr}_{B}(\mathrm{~g})$ może być przedstawiony w następującej postaci:

$$
W_{B i b r}(z a k r)=\left\langle E_{B W i b r}(z a k r),\left\{Y_{B W i b r}(z a k r)\right\}\right\rangle
$$

gdzie:

- $E_{B W i b r}(z a k r)$ - zbiór wprowadzanych uszkodzeń elementów;

- $Y_{B W i b r}(z a k r)$ - zbiór drganiowych sygnałów diagnostycznych;

- zakr - zakres pracy obiektu.

Eksperyment operacyjny przeprowadza się na obiekcie rzeczywistym o nieznanym stanie technicznym.

$$
W_{i b r_{O}}(z a k r)=\left\langle e_{\text {OWibr }}^{?}(z a k r), Y_{\text {OWibr }}(z a k r)\right\rangle
$$

Jeżeli uzyskane wyniki badań diagnostycznych można uzgodnić z posiadaną bazą wiedzy, tzn.:

$$
Y_{\text {OWibr }}(z a k r) \subseteq\left\{Y_{B W i b r}(z a k r)\right\}
$$

to określany jest bieżący stan obiektu

$$
e_{\text {OWibr }}^{?}(z a k r)=\left(e_{B W i b r}^{i}(z a k r) \in E_{B W i b r}(z a k r)\right)
$$

W przeciwnym przypadku, stan obiektu pozostaje nieznany i należy zastosować metodologię eksperymentu zespolonego.

Eksperyment zespolony składa się z dwóch faz: operacyjnej i badawczej, przy czym obligatoryjna jest faza operacyjna. Jeżeli symptomy $z$ bieżącego badania diagnostycznego są zgodne z dotychczasową wiedzą - identyfikowany jest stan obiektu. W przeciwnym przypadku uruchamiana jest faza badawcza, w ramach której następuje aktualizacja wiedzy diagnostycznej z wykorzystaniem informacji z fazy operacyjnej, procesu odnowy, bezpośrednich metod oceny stanu (włącznie z kontrolnym demontażem) oraz badań symulacyjnych.

$$
W i b r_{Z}(z a k r)=\left\langle e_{Z W i b r}^{*}(z a k r), Y_{Z W i b r}(z a k r)\right\rangle
$$

Jeżeli spełniona jest zależność:

$$
Y_{Z W i b r}(z a k r) \subseteq\left\{Y_{B W i b r}(z a k r)\right\}
$$

to bieżący stan jest elementem zbioru znanych stanów:

$$
e_{Z W i b r}^{*}(z a k r)=\left(e_{B W i b r}^{i}(z a k r) \in E_{B W i b r}(z a k r)\right)
$$

jeżeli nie - uruchamiana jest procedura aktualizacji bazy wiedzy:

$$
\begin{gathered}
\left\{Y_{B W i b r}(z a k r)\right\}^{\prime}=\left\{Y_{B W i b r}(z a k r)\right\} \cup Y_{\text {ZWibr }}(z a k r) \\
E_{B W i b r}^{\prime}(z a k r)=E_{B W i b r}(z a k r) \cup e_{\text {ZWibr }}^{*}(z a k r)
\end{gathered}
$$


W rezultacie zwiększa się liczność zbióru uszkodzeń oraz zbioru realizacji sygnałów diagnostycznych zawartych w bazie wiedzy.

Przykładem jest eksperyment przeprowadzony w ramach projektu badawczego, który obejmował zagadnienia związane z kompleksowym diagnozowaniem układu łożyskowania turbinowego silnika śmigłowcowego Allison 250-C20B [6].

\section{Parametryzacja sygnałów diagnostycznych $z$ wykorzystaniem transformaty Wigner-Ville'a}

Wynikiem badań diagnostycznych są zbiory pierwotnych sygnałów, które powinny być przetworzone do postaci umożliwiającej skuteczne wnioskowanie diagnostyczne $[6,12,13]$. W tym celu przeprowadza się parametryzację przebiegów czasowych polegającą na znalezieniu reprezentacji $\mathrm{w}$ postaci niewielkiego zbioru parametrów, przy zachowaniu informacji diagnostycznej niezbędnej w procesie wnioskowania $[3,6,10]$.

Do sygnałów wibroakustycznych generowanych przez maszyny wirnikowe stosuje się transformacje do dziedziny częstotliwości, a także czas-częstotliwość i czasskala. W niniejszej pracy parametryzację sygnałów wibroakustycznych przeprowadzono z wykorzystaniem transformaty Wigner-Ville'a [1, 2].

Transformacja Wigner-Ville'a generuje czasowo-częstotliwościową reprezentację sygnałów w postaci rozkładu energii sygnału względem dwóch zmiennych: czasu i częstotliwości. Energia sygnału $x$ może być wyznaczona jako kwadrat modułu sygnału lub jego transformaty Fouriera [2] :

$$
E_{x}=\int_{-\infty}^{+\infty}|x(t)|^{2} d t=\int_{-\infty}^{+\infty}|X(\omega)|^{2} d \omega
$$

Wprowadzając łączną gęstość energii w czasie i częstotliwości można zapisać [2]

$$
E_{x}=\int_{-\infty}^{+\infty} \int_{-\infty}^{+\infty} \rho(t, \omega) d t d \omega
$$

Rozkład Wigner-Ville'a (WVD) definiowany jest następująco [2]:

$$
W_{x}(t, \omega)=\int_{-\infty}^{+\infty} x(t+\tau / 2) x^{*}(t-\tau / 2) e^{-i 2 \pi \omega \tau} d \tau
$$

lub

$$
W_{x}(t, \omega)=\int_{-\infty}^{+\infty} X(\omega+\xi / 2) x^{*}(t-\xi / 2) e^{i 2 \pi \xi \tau} d \xi
$$


Synthesis of a multi-valued diagnostic model using the Wigner-Ville transformation Synteza wielowartościowego modelu diagnostycznego z wykorzystaniem...

\section{Wlaściwości rozkładu WVD}

Zachowanie energii [2]

$$
E_{x}=\int_{-\infty}^{+\infty} \int_{-\infty}^{+\infty} W_{x}(t, \omega) d t d \omega
$$

Własności brzegowe [2]

$$
\begin{aligned}
& \int_{-\infty}^{+\infty} W_{x}(t, \omega) d t=|X(\omega)|^{2} \\
& \int_{-\infty}^{+\infty} W_{x}(t, \omega) d \omega=|x(t)|^{2}
\end{aligned}
$$

Przyjmuje wartości ze zbioru liczb rzeczywistych [2]

$$
W_{x}(t, \omega) \in \mathfrak{R}, \forall t, \omega
$$

Twierdzenie o przesunięciu [2]

$$
\begin{aligned}
& y(t)=x\left(t-t_{0}\right) \Rightarrow W_{y}(t, \omega)=W_{x}\left(t-t_{0}, \omega\right) \\
& y(t)=x(t) e^{j 2 \pi \omega_{0} t} \Rightarrow W_{y}(t, \omega)=W_{x}\left(t, \omega-\omega_{0}\right)
\end{aligned}
$$

Skalowanie [2]

$$
y(t)=\sqrt{k} x(k t) ; k>0 \Rightarrow W_{y}(t, \omega)=W_{x}\left(k t, \frac{\omega}{k}\right)
$$

Filtrowanie [2]

$$
y(t)=\int_{-\infty}^{+\infty} h(t-s) x(s) \mathrm{ds} \Rightarrow \int_{-\infty}^{+\infty} W_{h}(t-s, \omega) W_{x}(\mathrm{~s}, \omega) \mathrm{d} s
$$

Modulacja [2]

$$
y(t)=m(t) x(t) \Rightarrow W_{y}(t, \omega)=\int_{-\infty}^{+\infty} W_{m}(t, \omega-\xi) W_{x}(\mathrm{~s}, \xi) \mathrm{d} \xi
$$

Praktyczne wykorzystanie transformaty Wigner-Ville'a wymaga zastosowania odpowiedniego oprogramowania. W pracy wykorzystano Time-Frequecy Toolbox (TFTb) dla środowiska Matlab [16] opracowany przez zespoły z Francji (CNRS Centre National de la Recherche Scientifique ) i USA (Rice University) [1, 2].

Poniżej przedstawiono nagłówek funkcji realizującej transformację Wigner- Ville’a badanego sygnału [2]. 


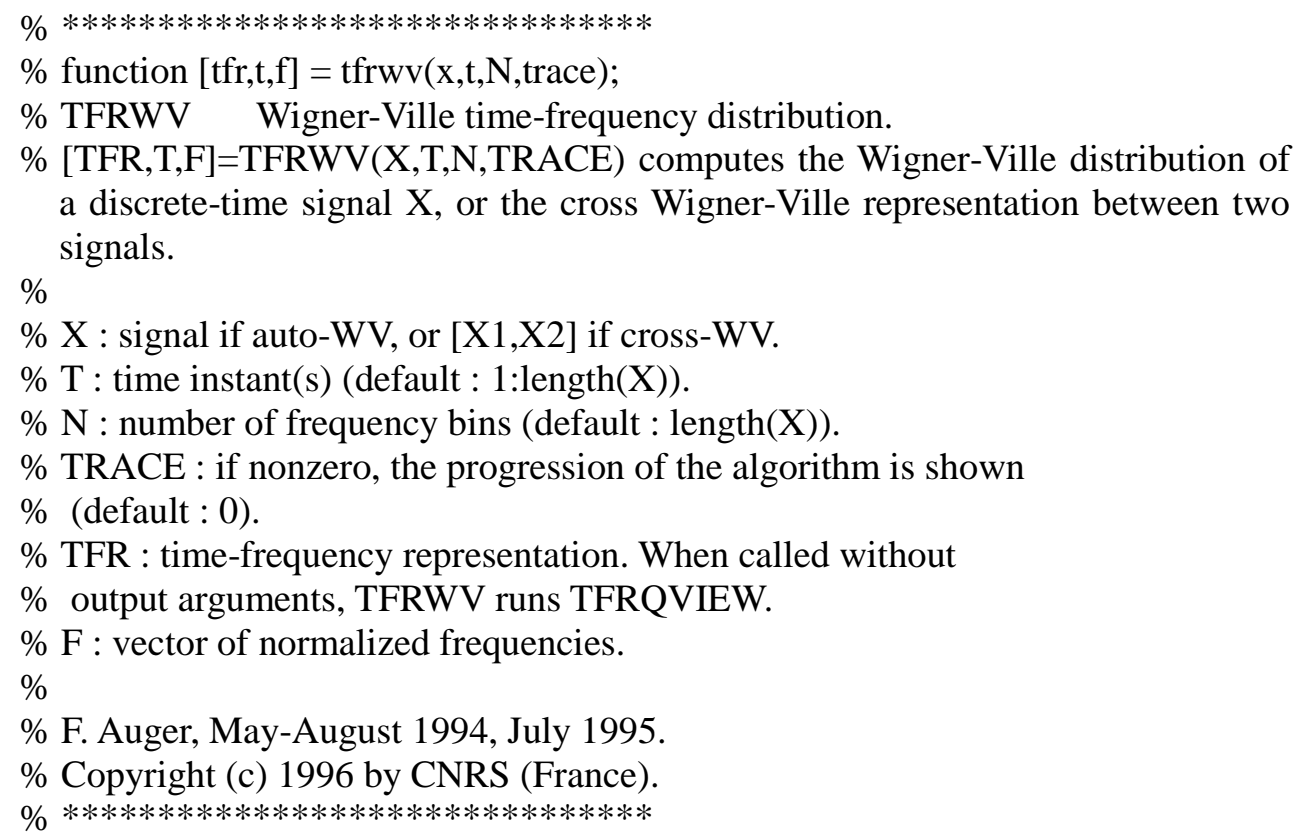

Argumentami wejściowymi są wektory: $x$ - próbek analizowanego sygnału i $t$ - chwil czasu pobrania próbek (o liczności $N x$ ) oraz założona częstotliwościowa rozdzielczośc $N<=N x$.

Argumentami wyjściowymi są: macierz tfr współczynników transformaty WignerVille'a o wymiarach $[N, N x]$ oraz wektor $f$ częstotliwości znormalizowanych względem częstotliwości próbkowania $f s$.

Otrzymane rezultaty stanowią podstawę wyznaczania wielowartościowej reprezentacji transformaty WV w celu implementacji w modelu diagnostycznym.

\section{Wielowartościowa reprezentacja transformaty Wigner-Ville’a}

Algorytm proponowanej metody wyznaczania wielowartościowej reprezentacji transformaty WV składa się z następujących etapów:

- wyznaczenie transformaty Wigner-Ville'a analizowanego sygnału (z wykorzystaniem przedstawionej wyżej funkcji $t f r w v$ ) ;

- obliczenie wartości skutecznych współczynników transformaty Wigner-Ville’a dla zbioru częstotliwości $F$ uwzględnionych w pierwotnej macierzy WV;

- wyznaczenie zbioru istotnych częstotliwości Fi na podstawie lokalnych maksimów wartości skutecznych współczynników transformaty WV;

- przeprowadzenie wielowartościowego kodowania wartości skutecznych współczynników transformaty WV odpowiadających częstotliwościom istotnym Fi. 
Synthesis of a multi-valued diagnostic model using the Wigner-Ville transformation Synteza wielowartościowego modelu diagnostycznego z wykorzystaniem...

Głowne elementy algorytmu ilustruje poniższy pseudo-kod:

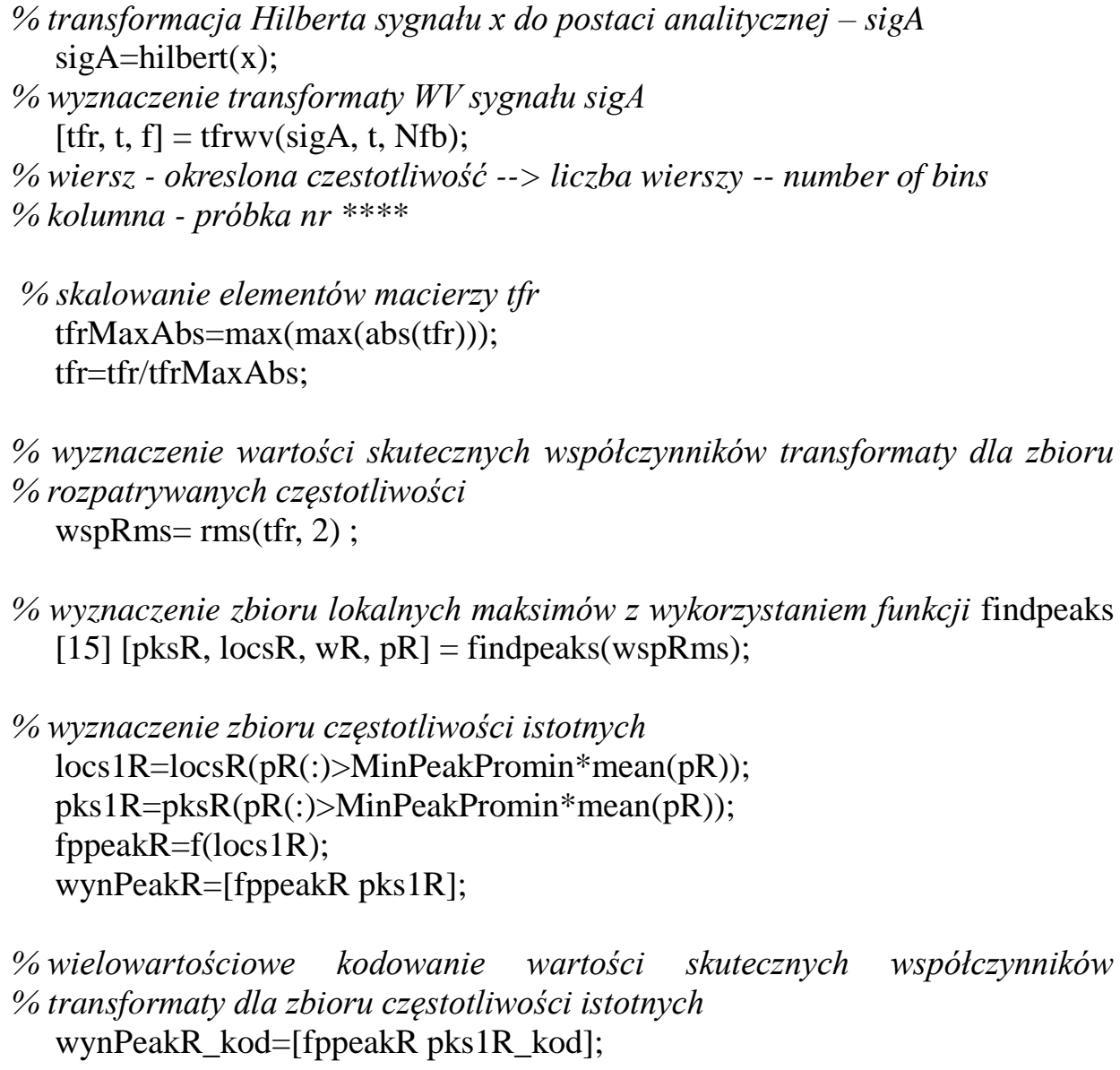

Kodowanie wielowartościowe polega na przypisaniu wartościom wspólczynników z określonego przedziału liczb rzeczywistych jednej liczby całkowitej ze znakiem. Zakresy zmienności współczynników należy podzielić na przedziały w taki sposób, aby $\mathrm{z}$ jednej strony zapewnić jak najwyższą rozdzielczość (rozróżnialność stanów/uszkodzeń), a z drugiej - zminimalizować liczbę błędnych diagnoz [4, 5]. Wyznaczanie przedziałów można rozpatrywać jako zagadnienie formalne klasteryzacji danych lub heurystyczne. W pierwszym przybliżeniu najczęściej stosuje się podejście heurystyczne wykorzystujące wiedzę ekspercką i nie wymagające dużego zbioru danych statystycznych. 
$\mathrm{W}$ procesie rozwoju systemu diagnostycznego, po zgromadzeniu odpowiedniej ilości materiału eksperymentalnego, wstępnie wyznaczone przedziały poddawane są okresowej aktualizacji z zastosowaniem metod formalnych [4, 5].

\section{Przykład analizy sygnału}

Analizie poddano sygnał drganiowy (oznaczony LL09A-2) zmierzony na obiekcie rzeczywistym.

Rys. 1 przedstawia przebieg czasowy sygnału, gęstość widmową energii oraz wykres 3D transformaty Wigner-Ville'a. Na rys. 2 przedstawiono:

- wartości skuteczne współczynników transformaty WV dla wszystkich częstotliwości uwzględnionych w transformacie tfr (linia ciągła);

- zbiór częstotliwości istotnych - wykres prążkowy (stem).

Wyniki wielowartościowego kodowania wartości skutecznych współczynników transformaty WV dla częstotliwości istotnych przedstawiono na rys. 3.
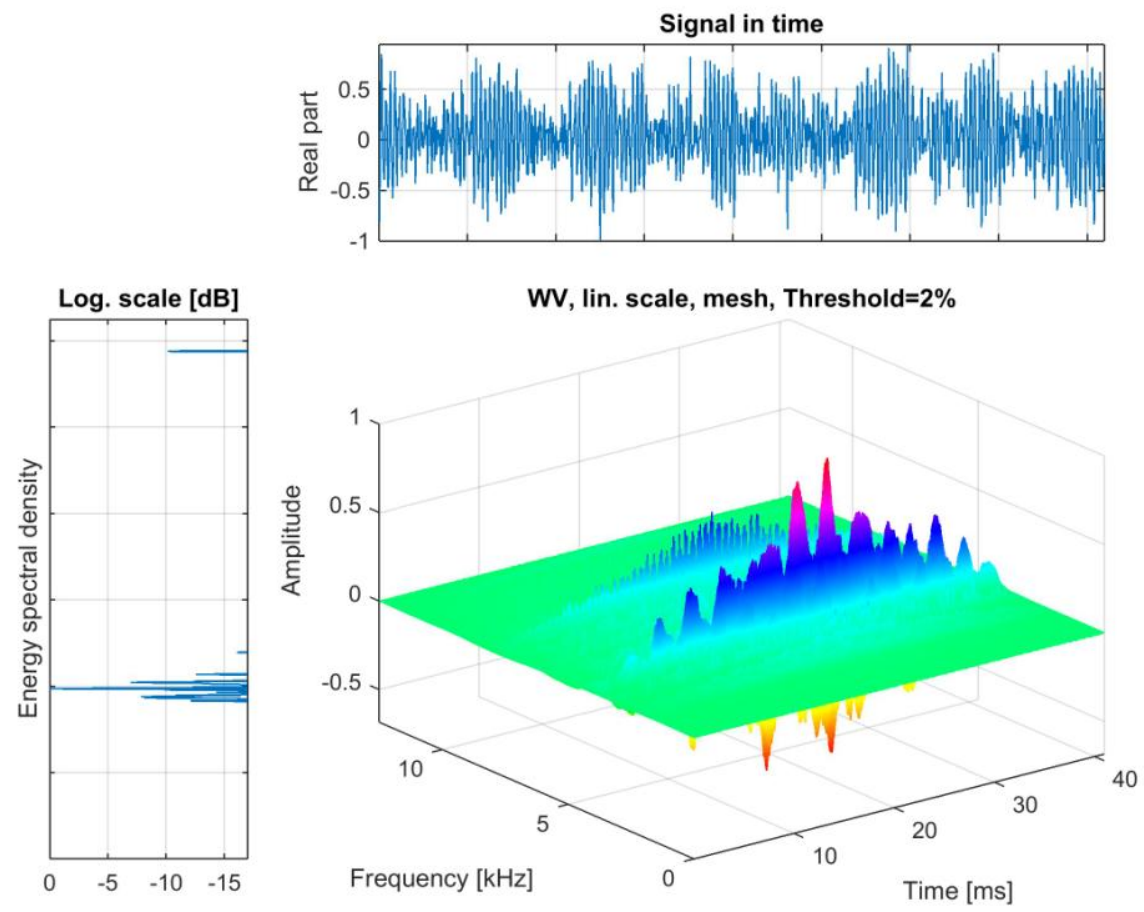

Rys. 1 Prezentacja graficzna transformaty Wigner-Ville'a analizowanego sygnału 
Synthesis of a multi-valued diagnostic model using the Wigner-Ville transformation Synteza wielowartościowego modelu diagnostycznego z wykorzystaniem...

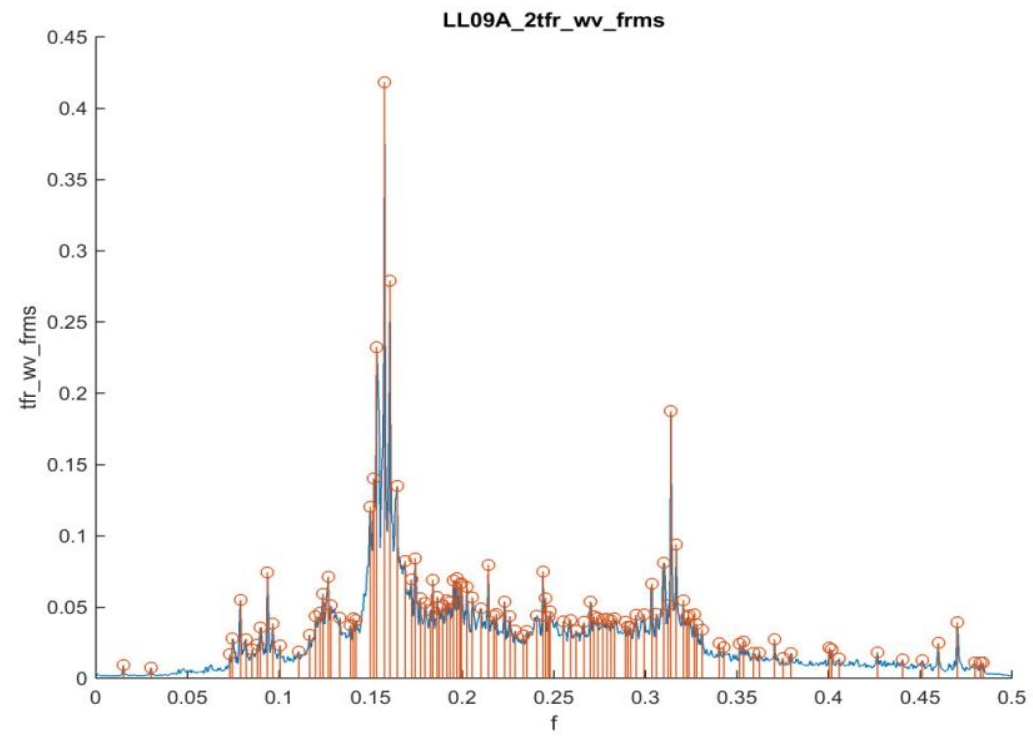

Rys. 2 Prezentacja graficzna zbioru częstotliwości istotnych analizowanego sygnatu

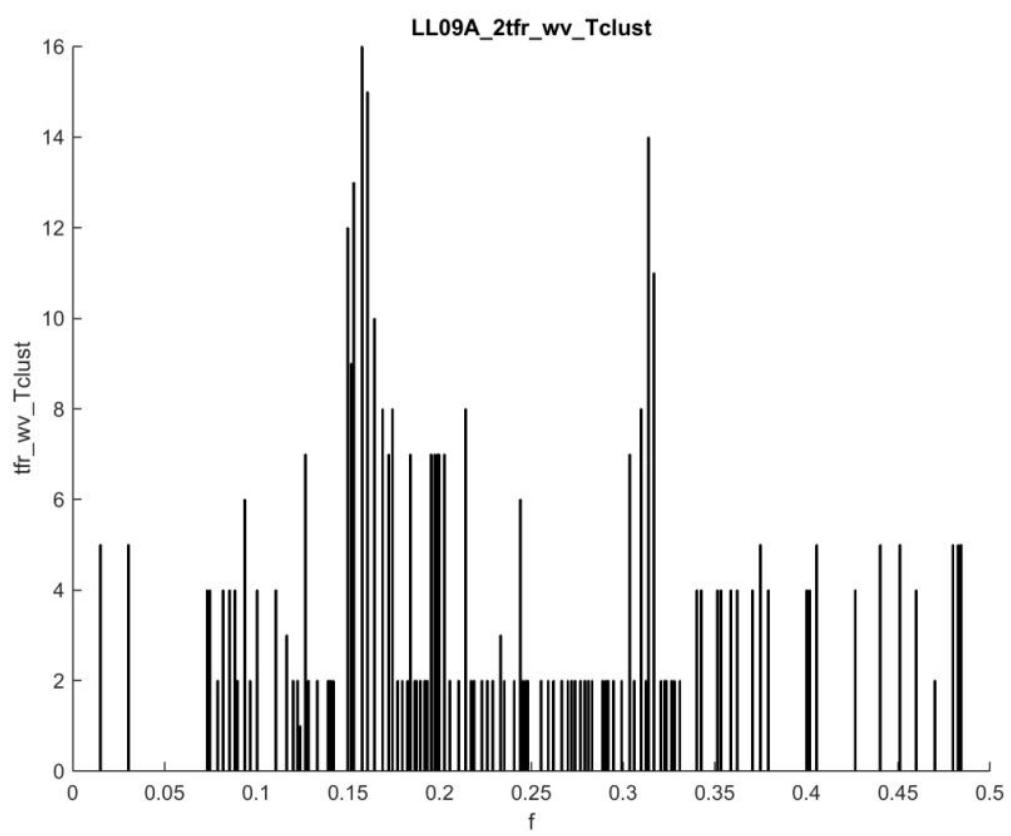

Rys. 3 Wielowartościowa reprezentacja zbioru częstotliwości istotnych analizowanego sygnatu 


\section{Podsumowanie}

W pracy przedstawiono zagadnienie syntezy modelu diagnostycznego z wykorzystaniem przekształcenia Wigner-Ville’a sygnałów wibroakustycznych zmierzonych na obiekcie rzeczywistym.

Ponieważ wnioskowanie diagnostyczne opiera się nie tyle na wartościach punktowych charakterystyk, co na ich przynależności do określonych przedziałów zastosowano kodowanie wielowartościowe. Wyniki analizy sygnałów przedstawione $\mathrm{w}$ postaci kodów umożliwiają porównywanie informacji diagnostycznej uzyskanej z transformaty Wigner-Ville'a i śledzenie zmiany stanu technicznego obiektu w czasie eksploatacji.

Rezultaty pracy mogą być wykorzystane w ekspertowych systemach diagnostycznych, zapewniających automatyzację przeprowadzanych analiz.

\section{Literatura}

[1] Auger, F., et al., Time-Frequency Toolbox. Reference Guide( CNRS - France, Rice University -USA). 1995-1996.

[2] Auger, F., et al., Time-Frequency Toolbox. User's Guide( CNRS - France, Rice University - USA). 1995-1996.

[3] Borowczyk, H., Ekspertowy system wspomagania wnioskowania diagnostycznego. W: Borowczyk H.. (red.) Problemy kompleksowego diagnozowania układu łożyskowania turbinowego silnika śmigłowcowego. Wyd. ITWL. Warszawa 2011. s. 147-163.

[4] Borowczyk, H., Elementy kompleksowej diagnostyki złożonych obiektów technicznych (monografia w przygotowaniu do druku).

[5] Borowczyk, H., A Multi-Valued Diagnostic Model Synthesis Based on Descrete Wavelet Transform, Journal of KONBiN 1 (17) 2011.https://doi.org/10.2478/jok-2013-0104.

[6] Borowczyk, H., ed. Problemy kompleksowego diagnozowania układu łożyskowania turbinowego silnika śmigłowcowego. Wyd. ITWL. Warszawa $2011163 \mathrm{~s}$.

[7] Borowczyk, H., P. Lindstedt, and J. Magier, Układ łożyskowania turbinowego silnika śmigłowcowego jako obiekt diagnozowania. W : Borowczyk H.. (red.) Problemy kompleksowego diagnozowania układu łożyskowania turbinowego silnika śmigłowcowego. Wyd. ITWL. Warszawa 2011. s. 21-30.

[8] Cempel, C., Podstawy wibroakustycznej diagnostyki maszyn, WNT, Warszawa 1982.

[9] Cempel, C., Wibroakustyka stosowana, WNT, Warszawa 1989.

[10] Cholewa, W., Data processing and reasoning in technical diagnostics. WNT, Warszawa, 1995.

[11] Korbicz, J., Diagnostyka procesów. Modele. Metody sztucznej inteligencji. Zastosowania. WNT, Warszawa 2002.

[12] Lindstedt, P., Praktyczna diagnostyka maszyn i jej teoretyczne podstawy. Wyd. Naukowe ASKON, Warszawa 2002. 
Synthesis of a multi-valued diagnostic model using the Wigner-Ville transformation Synteza wielowartościowego modelu diagnostycznego z wykorzystaniem...

[13] Lindstedt, P. and H. Borowczyk, Kompleksowy system diagnostyczny statku powietrznego, w: Problemy Badań i Eksploatacji Techniki Lotniczej, tom 4, rozdz. 7, Wyd. ITWL, Warszawa 1999.

[14] Lindstedt, P., H. Borowczyk, and J. Magier, Diagnostyka silnika śmigłowcowego w inżynieryjno-lotniczym otoczeniu, Prace Naukowe ITWL, zeszyt nr 17. Wyd. ITWL, Warszawa 2003.

[15] MathWorks. findpeaks - help. Available from: www.mathworks.com/help/signal/ref/findpeaks.html?s_tid=doc_ta.

[16] MathWorks. Help Available from: https://www.mathworks.com/help/.

[17] Niziński, S., Elementy eksploatacji obiektów technicznych, Wyd. Uniwersytetu Warmińsko-Mazurskiego, Olsztyn 2000.

[18] Spychała, J., M. Szczekala, and M. Żokowski, Diagnozowanie w dziedzinie wibroakustycznej. W: Borowczyk H. (red.) Problemy kompleksowego diagnozowania układu łożyskowania turbinowego silnika śmigłowcowego. Rozdz. 5. Wyd. ITWL Warszawa 2011.

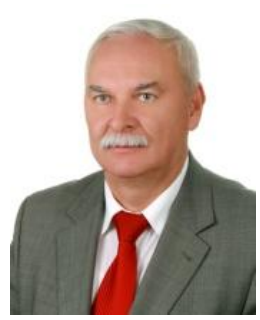

PhD Eng. Henryk Borowczyk, Aircraft Engine Division, Air Force Institute of Technologyl, Warsaw.

Research activities: Comprehensive diagnostics of turbine aircraft engines using information theory, mathematical identification methods of dynamic systems and artificial intelligence. 